\title{
Polarizable Ions at Interfaces
}

\author{
Yan Levin \\ Instituto de Física, Universidade Federal do Rio Grande do Sul, Caixa Postal 15051, CEP 91501-970, Porto Alegre, RS, Brazil
}

(Received 17 December 2008; published 8 April 2009)

\begin{abstract}
A nonperturbative theory is presented which allows us to calculate the solvation free energy of polarizable ions near water-vapor and water-oil interfaces. The theory predicts that larger halogen anions are adsorbed at the interface, while the alkali metal cations are repelled from it. The density profiles calculated theoretically are similar to those obtained using molecular dynamics simulations with polarizable force fields.
\end{abstract}

DOI: 10.1103/PhysRevLett.102.147803

PACS numbers: 61.20.Qg, 05.20.-y, 82.45.Gj

There are a number of long standing mysteries in the fields of physical chemistry and biophysics. The Hofmeister effect [1], which has now been known for over 120 years is, perhaps, one of the oldest and most puzzling ones. Hofmeister observed that different ions have a very different effect on the stability of protein solutions. While some electrolytes are very efficient at salting out proteins, others lead to protein precipitation only at much larger concentrations. A related mystery, which is also very old, has to do with the surface tensions. Some hundred years ago Heydweiller [2] noted that adding a strong electrolyte to water leads to an increase in the surface tension of the water-air interface. While the dependence on the type of cation is weak, there is a strong variation of the excess surface tension with the type of anion-the lighter halides lead to larger excess surface tension than the heavier ones. The sequence is precisely the reverse of the Hofmeister one. Both effects are completely unaccounted for by the current theories of electrolytes, which go back to the pioneering work of Debye and Hückel (DH) [3,4] and Onsager and Samaras (OS) [5-7].

Some clues to the failure of the DH and the OS theories started to appear in the 1990s when the photoelectron emission experiments [8] and molecular dynamics simulations with polarizable force fields showed that contrary to the common wisdom and the predictions of OS theory, there were ions present at the air-water interface $[9,10]$. The simulations found that while hard alkali metal ions such as potassium and sodium and small halides such as fluoride [11] are repelled from the interface, the softer more polarizable anions such as bromide and iodine are actually attracted to it [12]. Presence of highly reactive halogens at the air-water interface of aerosol particles might help to explain the excessive rate of ozone depletion [13].

In this Letter a new class of electrolyte models will be introduced. Unlike the previous approaches, the polarizability of ions will be explicitly taken into account. The new theory is intrinsically nonperturbative-all the moments of the ionic charge distribution, and not just the dipole, are taken into account. The calculated solvation free energies are used to obtain the interaction potential of polarizable ions with an interface and to calculate the anion and cation density profiles.

Since the pioneering work of Debye and Hückel, ions have been modeled as hard spheres with a point charge located at the center [4]. While perfectly reasonable for describing bulk properties of electrolytes, this approach is bound to fail when applied to polarizable ions near a dielectric interface. The reason for this is easily understood by considering the simplest model of a perfectly polarizable ion idealized as a conducting spherical shell with a mobile surface charge. When such an ion moves across a dielectric air- (oil-)water interface, the charge on its surface shifts progressively from the exposed air or oil portion to the part that still remains hydrated. For perfectly polarizable ions, energy cost of charge localization is very low and is easily compensated by the decrease in the cavitational energy [14] as the ion moves across the interface.

To make the discussion quantitative, consider a polarizable ion-modeled as a conducting sphere of radius $a$ and charge $q$, see Fig. 1-at an air- (oil)-water interface. Both half-spaces will be treated as dielectric continuums with permittivities $\epsilon_{w}$ and $\epsilon_{o}$ for water and air (oil), respectively. To gain insight into the problem we first consider an ion with one of its hemispheres submerged in water and the other exposed to air, $h=a$, Fig. 1. This problem can be solved exactly, yielding a purely radial electric field and the electrostatic self energy of the ion given by

$$
U_{s}(a)=\frac{q^{2}}{\left(\epsilon_{w}+\epsilon_{o}\right) a} .
$$

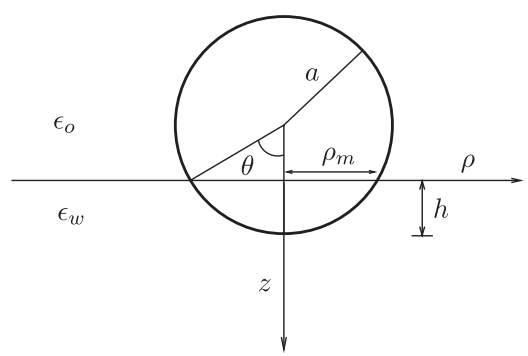

FIG. 1. Ion of radius $a$ at an interface. 
We can also calculate the ratio of the surface charge on the two hemispheres, obtaining $q_{o} / q_{w}=\epsilon_{o} / \epsilon_{w}$. This means that for an air-water interface with $\epsilon_{o} / \epsilon_{w}=1 / 80$, the fraction of the ionic charge located in air is only $1 \%$ of the total charge. For a perfectly polarizable ion half exposed to air, $99 \%$ of its charge remains hydrated. This is very different from nonpolarizable ions, which under the same conditions will have half of their charge exposed to the low dielectric environment, at a huge electrostatic self energy cost. This is the reason why nonpolarizable ions cannot penetrate into the interfacial region. Evidently this is not the case for polarizable ions which can adjust their charge distribution to minimize the electrostatic self energy cost. Unfortunately, once we leave the symmetric case of an ion located half way across the interface, no exact solution is possible and approximate methods must be used.

The self energy of a perfectly polarizable ion with its lower extreme situated a distance $h$ from the interface, see Fig. 1, can be written as,

$$
U_{s}(h)=\frac{q^{2}}{2 \epsilon_{w} C},
$$

where the capacitance $C=a f\left(\epsilon_{o} / \epsilon_{w}, h / a\right)$ and $f(x, y)$ is a scaling function. In the limit $\epsilon_{o} \ll \epsilon_{w}$, we can expand $f$ in powers of $\epsilon_{o} / \epsilon_{w}$,

$$
f\left(\frac{\epsilon_{o}}{\epsilon_{w}}, \frac{h}{a}\right)=f\left(0, \frac{h}{a}\right)+\frac{\epsilon_{o}}{\epsilon_{w}} f_{1}\left(\frac{h}{a}\right) .
$$

The value of the scaling function $f(0, h / a)$ determines the capacitance in the limit of vanishing air permittivity. In this limit the electric field lines originating on the charge inside water must be tangential to the interface, so that the normal component of the electric field vanishes. Even this problem, however, is difficult to solve analytically. Exact solution is possible, however, when $h / a \ll 1$. In this limit all charge is confined to a small spherical cap located inside water. The curvature effects can be neglected, and the problem reduces to finding the solution of a mixed boundary value problem in cylindrical coordinates: $\nabla^{2} \phi(z, \rho)=$ $0, \phi^{\prime}(0, \rho)=0$ for $\rho>\rho_{m}$, and $\phi(0, \rho)=V$ for $\rho \leq \rho_{m}$, where prime refers to the derivative with respect to $z, V$ is the electrostatic potential of the spherical cap, and $\rho_{m}$ is its radius, see Fig. 1. Mixed boundary value problems are notoriously difficult to study. Fortunately, this particular one can be solved analytically using the Hankel transform techniques [15]. We find

$$
\phi(z, \rho)=\frac{2 V}{\pi} \int_{0}^{\infty} d k \frac{\sin \left(k \rho_{m}\right)}{k} J_{0}(k \rho) e^{-k z} .
$$

The capacitance of the spherical cap can now be calculated to be $C_{c}=\rho_{m} / \pi$. We note that this is just half the value of the capacitance of a disk of radius $\rho_{m}$ in vacuum. This result can be understood by considering a charged disk in front of a dielectric medium of very low permittivity. The image charge induced on the interface will then be of the same sign as on the disk, and in the limit $\epsilon_{o} \ll \epsilon_{w}$ it will also be the same in magnitude. Thus, one needs only half the charge of the disk in vacuum to have the same potential.

In view of the natural symmetry of the problem it is convenient to express $C_{c}$ in terms of the angle variable $\theta$, so that $C_{c}=a \theta / \pi$ and $f(0, h / a)=\theta / \pi$. Writing the capacitance in terms of $\theta$ extend the range of validity of $C_{c}$ outside the limit of completely flat disc to larger spherical caps. In particular, for a particle located half way across the interface, $h=a, \theta=\pi / 2$, we find $f(0,1)=1 / 2$, which agrees precisely with the exact result of Eq. (1). Furthermore, comparing Eqs. (2) and (3) with Eq. (1), we see that $f_{1}(1)=1 / 2$. We are now in position to write an approximate expression for the self energy of a perfectly polarizable ion moving across a dielectric interface,

$$
U_{s}(h)=\frac{q^{2}}{2 \epsilon_{w} a} \frac{1}{\frac{\theta(h)}{\pi}+\frac{\epsilon_{o}}{2 \epsilon_{w}}} .
$$

where $\theta(h)=\operatorname{Re}\left[\arccos \left(1-\frac{h}{a}\right)\right]$. The real part of $\arccos (x)$ is used in order to continue its validity into the regions $h>$ $2 a$, where $\theta(h)=\pi$. For $h>2 a$ the electrostatic self energy reduces to $U_{s} \approx q^{2} / 2 \epsilon_{w} a$, which is the usual Born self energy of a bulk ion. In writing Eq. (5), we have approximated the scaling function $f_{1}(x)$ by a constant, $f_{1}(x)=1 / 2$. This is permissible, since when the ratio $\epsilon_{o} / \epsilon_{w} \ll 1$, the prefactor of $f_{1}$ is very small and the precise value of $f_{1}(x)$ is not important-it is completely dominated by the first term of Eq. (3). We should note, however, that although Eq. (5) is very accurate for $\pi \epsilon_{o} /\left(2 \epsilon_{w}\right)<\theta<3 \pi / 4$, and for $h / a \gg 1$, it does not describe perfectly the crossover from the interfacial to the bulk regime. The reason for this is that Eq. (5) does not fully account for the image contribution to the electrostatic energy at distances $h>a$. It is possible to include this corrections into the theory at the expense of more complicated expressions. In practice, however, we note that the image contribution is screened very strongly [6], with the characteristic length equal to half the Debye length $\xi_{D}, U_{\mathrm{im}}(z) \approx q^{2} \exp \left(-2 z / \xi_{D}\right) /\left(4 \epsilon_{w} z\right)$. Therefore, for concentrations of electrolyte above physiological ones $150 \mathrm{mM}$, the image contribution decays to zero after only a few angstroms. For now, we shall, therefore, ignore the image effect in the crossover region.

The force that drives ions towards the interface arises from the cavitational energy. Presence of ions disturbs the hydrogen bond network of water and costs energy. We can estimate this energy cost by considering a cavity which must be formed in water to accommodate an ion. For small cavities of radius $a<4 \AA$, which do not significantly perturb the hydrogen bonds, the energy cost scales with the volume of the void, while for larger cavities the energy cost scales with the cavity surface area [16]. This is, the so called, hydrophobic crossover from small to large length scales [17]. Small alkali metal and halogen ions are in the volumetric scaling regime. If one part of an ion leaves the aqueous environment, the cavitational energy will decrease 
proportionately to the volume exposed. This results in a short range cavitation potential which forces ions to move into the air (oil) phase,

$$
U_{\text {cav }}(h)=\left\{\begin{array}{ll}
\nu a^{3} & \text { for } h \geq 2 a \\
\frac{1}{4} \nu a^{3}\left(\frac{h}{a}\right)^{2}\left(3-\frac{h}{a}\right) & \text { for } 0<h<2 a
\end{array} .\right.
$$

From the results of bulk simulations [14], we calculate that $\nu \approx 0.3 k_{B} T / \AA^{3}$. To account for the fact that a cavity containing an ion should be somewhat larger than the ionic crystallographic size, we will use $\nu \approx 0.5 k_{B} T / \AA^{3}$. This corresponds to the cavity radius about $20 \%$ larger than the crystallographic radius.

For small nonpolarizable ions, the cavitational energy is not sufficient to force ions into the low dielectric environment-the electrostatic energy cost is way too large. On the other hand, for soft polarizable ions, the electrostatic self energy cost is very small, since the ionic charge distribution can easily deform to remain mostly within the hydrated portion of the ion. The significant gain in the cavitational energy, and the low cost in electrostatic self energy, makes it energetically favorable for polarizable ions to move into the interfacial region. The amphiphilic nature of large ions, such as hexafluorophosphate $P F_{6}^{-}$, has been known for a long time. The cavitational energy for these ions is so large, that they actually adsorb to the interface, lowering its surface tension [18]. What has been discovered recently is that smaller polarizable ions apparently can also have some amphiphilic activity-although not sufficiently large to lower the interfacial tension [8,12].

So far we have considered only perfectly polarizable ions. Real ions, however, have finite polarizability. It is not obvious how the effects of finite polarizability can be included in the formalism developed above. In fact it is not even clear, if the concept of bulk polarizability, as a linear response to the external field, is relevant in the interfacial geometry, where a rapid variation of the dielectric constant makes all the moments of the charge distribution-not just the dipole-relevant. For perfectly polarizable ions we have avoided this difficulty by solving the full electrostatics problem for a conducting sphere. For ions of finite polarizability, to have a completely quantitative picture it might be necessary to go to full $a b$ initio calculations. In the absence of such, we can still gain some insight into this difficult problem by considering a simple model. In the spirit of Landau, we will construct the polarization energy $U_{p}$ by exploiting the symmetries of the problem.

Consider an ion of radius $a$ and bulk polarizability $\gamma$. We will define the relative polarizability of this ion as $\alpha \equiv$ $\gamma / \gamma_{0}$, where $\gamma_{0}=a^{3}$ is the polarizability of an ideal ion of the same radius, modeled as a conducting sphere. For nonideal ions with $0 \leq \alpha<1$, the surface charge can not fully adjust to the external electric field. Therefore, for such ions, there is an additional nonelectrostatic_-quantum mechanical - energy cost for dislocating ionic charge from its position of equilibrium. Suppose that for a given ion the fraction of its total charge inside water is $x$, then the charge exposed to air (oil) will be $(1-x) q$. For ions which are highly polarizable $x \approx 1$, as long as $\theta$ is not too small. There is, however, a polarization energy cost for shifting a fraction of the ionic charge (assumed to be originally uniformly distributed along the surface of the ion) from its equilibrium position in the air portion of the ion to the water part. Within our simple dielectric model $U_{p}$ must be invariant under the transformation $q \rightarrow-q$. It must also be invariant under the transformation $\theta \rightarrow \pi-\theta$, when $\epsilon_{o} \leftrightarrow$ $\epsilon_{w}$, and $x \rightarrow 1-x$. To respect these symmetries, the polarization energy must be an even function of the difference between the initial (before exposure) and the final (after exposure) amount of charge on the part of the ion exposed to air or oil. Taking all these considerations into account and recalling Eq. (5) for a perfectly conducting sphere, we arrive at the polarization energy for a nonideal ion,

$$
\begin{aligned}
\beta U_{p}(h ; x)= & \frac{\lambda_{B}}{2 a}\left[\frac{\pi x^{2}}{\theta}+\frac{\pi(1-x)^{2} \epsilon_{w}}{\epsilon_{o}(\pi-\theta)}\right] \\
& +g\left[x-\frac{1-\cos (\theta)}{2}\right]^{2},
\end{aligned}
$$

where $\beta=1 / k_{B} T$ and $\lambda_{B}=q^{2} / \epsilon_{w} k_{B} T$ is the Bjerrum length in water. The terms in the first square brackets of Eq. (6) are the electrostatic self energy costs of the parts of the ion exposed to the water and the air, respectively. The second brackets contain the energy cost arising from the induced inhomogeneity in the ionic surface charge distribution. The coupling constant $g$ must be a function of the relative polarizability, $g(\alpha)$. In the limit $\alpha \rightarrow 1$, ion becomes perfectly polarizable, so that $g \rightarrow 0$; while in the limit $\alpha \rightarrow 0$, ion becomes completely hard and $g \rightarrow \infty$. To account for these, we will write $g(\alpha)=\chi(1-\alpha) / \alpha$, where $\chi$ is a pure number. The precise value of $\chi$ can only be obtained from the $a b$ initio calculations. For now we will take it to be of order unity, $\chi \approx 1$. Minimizing Eq. (6) with respect to $x$ gives the fraction of the total charge located on the hydrated part of the ion, $x_{\min }(h)$. Substituting this back into Eq. (6), yields the polarization potential that an ion feels as it moves across the interface $U_{p}(h)=U_{p}\left(h ; x_{\min }(h)\right)$. For an ideal ion of $\alpha=1$ located half way across the interface $h=a$, the energy $U_{p}(a)$ reduces precisely to the expression given by Eq. (1). Therefore, for this case, the formalism developed above gives the exact result. The total solvation potential felt by an ion of relative polarizability $\alpha$ is $U_{\text {sol }}(h ; \alpha)=$ $U_{\text {cav }}(h)+U_{p}(h)$. Using this potential, we can calculate the ionic density profiles inside a small water droplet of radius $R$ containing $N$ cations and $N$ anion, by explicitly solving a modified Poisson-Boltzmann equation,

$$
\begin{aligned}
\nabla^{2} \phi(r)= & \frac{q N}{\epsilon_{w}}\left(\frac{e^{\beta q \phi(r)-\beta U_{\mathrm{sol}}\left(h ; \alpha_{a}\right)}}{\int_{0}^{R} r^{2} d r e^{\beta q \phi(r)-\beta U_{\mathrm{sol}}\left(h ; \alpha_{a}\right)}}\right. \\
& \left.-\frac{e^{-\beta q \phi(r)-\beta U_{\mathrm{sol}}\left(h ; \alpha_{c}\right)}}{\int_{0}^{R} r^{2} d r e^{-\beta q \phi(r)-\beta U_{\mathrm{sol}}\left(h ; \alpha_{a}\right)}}\right),
\end{aligned}
$$



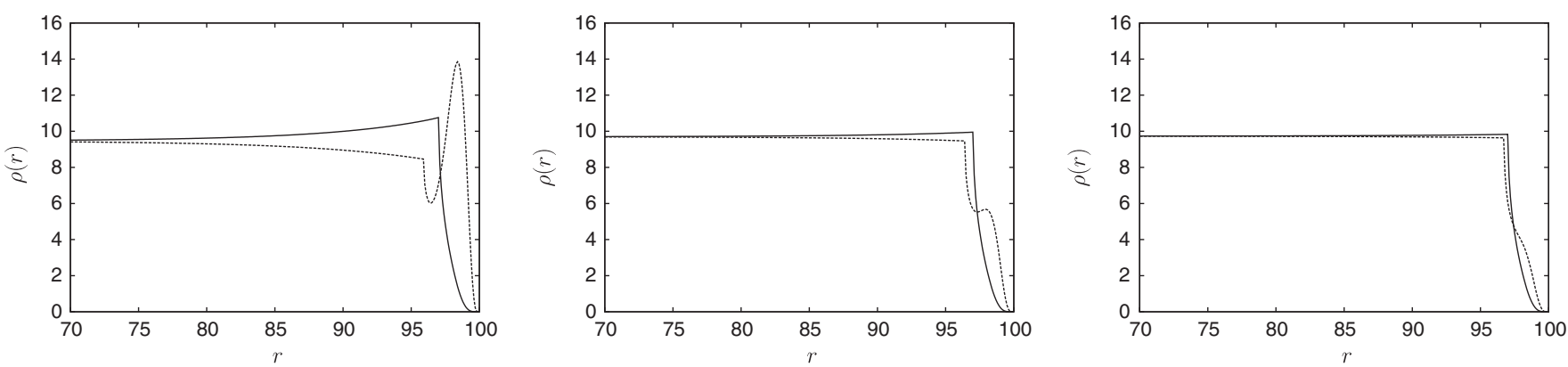

FIG. 2. Density profiles $\left(\times 10^{5}\right)$ of $\mathrm{KI}, \mathrm{KBr}$, and $\mathrm{KCl}$, from left to right. The radius of the water drop is $R=100 \AA$ and it contains $N_{+}=N_{-}=380$ ions at physiological concentration of $150 \mathrm{mM}$. Solid curve is for cation $(\mathrm{K})$ and the dashed ones are for anions. In all cases potassium is depleted from the interface, but there is a formation of a double layer, where the excess of anion directly at the interface results in a build up of potassium in its vicinity.

where $r$ is the distance measured from the center of the water droplet, $h=R-r$, and $\alpha_{c}$ and $\alpha_{a}$ are the relative polarizabilities of cations and anions, respectively. The density profiles for electrolyte solutions of $\mathrm{KI}, \mathrm{KBr}$, and $\mathrm{KCl}$ are presented in Fig. 2. The polarizabilities of ions $\gamma_{\mathrm{K}}=0.79 \AA^{3}, \quad \gamma_{\mathrm{I}}=7.4 \AA^{3}, \quad \gamma_{\mathrm{Br}}=5.07 \AA^{3}, \quad \gamma_{\mathrm{Cl}}=$ $3.77 \AA^{3}$, where take from Ref. [19] and the ionic sizes $a_{\mathrm{K}}=1.49 \AA, a_{\mathrm{I}}=2.05 \AA, a_{\mathrm{Br}}=1.8 \AA, a_{\mathrm{Cl}}=1.64 \AA$, from Ref. [20]. In agreement with the polarizable force fields simulations, the theory predicts that iodine is strongly adsorbed at the air-water interface. We also find that there is a significant concentration of bromide, while chloride, potassium and fluoride (not shown) are depleted from the interfacial region. The current theory, however, predicts that there is less halide ion adsorption than is found in the simulations. The difference might due to the overestimate of the neat water-vapor surface potential predicted by the polarizable force field simulations to be $-500 \mathrm{mV} \approx-20 k_{B} T / q$. Such a huge junction potential will irreversibly drive polarizable halides toward the vapor phase, resulting in a large density build up along the interface. Recent $a b$ initio simulations [21], however, find a much smaller contact potential, $-18 \mathrm{mV} \approx-0.7 k_{B} T / q$, for a water-vapor interface. This might lead to a smaller adsorption, in line with the predictions of the present theory. Finally, since the surface tension of electrolyte solution is directly related to ionic adsorption in the interfacial region, the theory developed also accounts for the Hofmeister series for halogens.

I am grateful to Felipe Rizzato and Alexandre P. dos Santos for help with numerics. This work is partially supported by CNPq, INCT-FCx, and the US-AFOSR under the Grant FA9550-06-1-0345.

[1] F. Hofmeister, Arch. Exp. Pathol. Pharmakol. 24, 247 (1888).

[2] A. Heydweiller, Ann. Phys. (Leipzig) 33, 145 (1910).
[3] P. W. Debye and E. Hückel, Phys. Z. 24, 185 (1923).

[4] Y. Levin, Rep. Prog. Phys. 65, 1577 (2002).

[5] C. Wagner, Phys. Z. 25, 474 (1924).

[6] L. Onsager and N. N. T. Samaras, J. Chem. Phys. 2, 528 (1934).

[7] Y. Levin, J. Chem. Phys. 113, 9722 (2000); Y. Levin and J. E. Flores-Mena, Europhys. Lett. 56, 187 (2001); V. S. Markin and A. G. Volkov, J. Phys. Chem. B 106, 11810 (2002); D. S. Dean and R. R. Horgan, Phys. Rev. E 69, 061603 (2004); W. Kunz, L. Belloni, O. Bernard, and B. W. Ninham J. Phys. Chem. B 108, 2398 (2004); A. Onuki, J. Chem. Phys. 128, 224704 (2008); M. Bier, J. Zwanikken, and R. van Roij, Phys. Rev. Lett. 101, 046104 (2008).

[8] G. Markovich, S. Pollack, R. Giniger, and O. Cheshnovsky, J. Chem. Phys. 95, 9416 (1991); S. Ghosal et al., Science 307, 563 (2005).

[9] L. X. Dang and D.E. Smith, J. Chem. Phys. 99, 6950 (1993).

[10] S. J. Stuart and B. J. Berne, J. Phys. Chem. A 103, 10300 (1999).

[11] M. A. Brown et al., Phys. Chem. Chem. Phys. 10, 4778 (2008).

[12] P. Jungwirth and D. J. Tobias, J. Phys. Chem. B 106, 6361 (2002); P. Jungwirth and D. J. Tobias, Chem. Rev. 106, 1259 (2006).

[13] E. M. Knipping, M. J. Lakin, K. L. Foster, P. Jungwirth, D. J. Tobias, R. B. Gerber, D. Dabdub, and B. J. FinlaysonPitts, Science 288, 301 (2000).

[14] S. Rajamani, T. M. Truskett, and S. Garde, Proc. Natl. Acad. Sci. U.S.A. 102, 9475 (2005).

[15] J.D. Jackson, Classical Electrodynamics (Wiley, New York, 1999).

[16] K. Lum, D. Chandler, and J. D. Weeks, J. Phys. Chem. B 103, 4570 (1999).

[17] D. Chandler, Nature (London) 417, 491 (2002).

[18] J. E. B. Randles, Discuss. Faraday Soc. 24, 194 (1957).

[19] N. C. Pyper, C. G. Pike, and P. P. Edwards, Mol. Phys. 76, 353 (1992).

[20] B. S. Gourary and F. S. Adrian, Solid State Phys. 10, 127 (1960).

[21] S. M. Kathmann, I.-F. W. Kuo, and C. J. Mundy, J. Am. Chem. Soc. 130, 16556 (2008). 